\title{
Discrete solvent effects on the effective interaction between charged colloids
}

\author{
E. Allahyarov and H. Löwen \\ Institut für Theoretische Physik II, Heinrich-Heine-Universität Düsseldorf, D-40225 Düsseldorf, Germany
}

(February 9, 2020)

\begin{abstract}
Using computer simulations of two charged colloidal spheres with their counterions in a hard sphere solvent, we show that the granular nature of the solvent significantly influences the effective colloidal interaction. For divalent counterions, the total effective force can become attractive generated by counterion hydration, while for monovalent counterions the forces are repulsive and well-described by a solvent-induced colloidal charge renormalization. Both effects are not contained in the traditional "primitive" approaches but can be accounted for in a solvent-averaged primitive model.
\end{abstract}

PACS: 82.70.Dd, 61.20.Ja

Supramolecular aggregates, such as colloids, polymers or biological macromolecules, are typically suspended in a molecular solvent which guarantees their stability and profoundly influences their viscoelastic properties [1]: examples range from paints to dense DNA-solutions in biological cells. A full "ab initio" statistical description of supramolecular solutions should start from a molecular scale including the solvent explicitly. Obviously this is a tremendous task due to the large length scale separation between the microscopic solvent and the mesoscopic solute and the enormous number of solvent particles which have to be considered explicitly. Therefore, most of the common statistical approaches are based on so-called "primitive" models insofar as they disregard the molecular nature of the solvent completely such that solvent properties only enter via a continuous background.

A particular example for such a separation of length scales are charged colloidal suspensions [2] consisting of highly charged mesoscopic particles (so-called polyions) suspended in water or any other organic solvent together with their oppositely charged microscopic counterions. The key quantity to understand the stability, structure and dynamics of such colloidal dispersions is the effective interaction between two polyions, as a function of their mutual distance $r$. Neglecting the discrete solvent this quantity has been calculated using modeling on different descending levels: i) the "primitive model" (PM) of strongly asymmetric electrolytes which takes into account explicitly the counterions ii) the nonlinear Poisson-Boltzmann approach which is inferior to the $\mathrm{PM}$ as it neglects counterion correlations, iii) the linearized screening theory resulting in a Yukawa form for the effective interaction potential as given by the electrostatic part of the celebrated Derjaguin-Landau-VerweyOverbeek (DLVO) theory [3]. The main effects of nonlinear Poisson-Boltzmann theory can be encaptured by a similar Yukawa potential but with "renormalized" parameters leading to the concept of colloidal charge renormalization [4. This picture is consistent with experimental data in dilute bulk solutions with monovalent counterions [5],6]. Very strong correlations between divalent and trivalent counterions, however, may lead to attractive effective forces between like-charge polyions as shown in recent computer simulations of the PM [7] 9 .

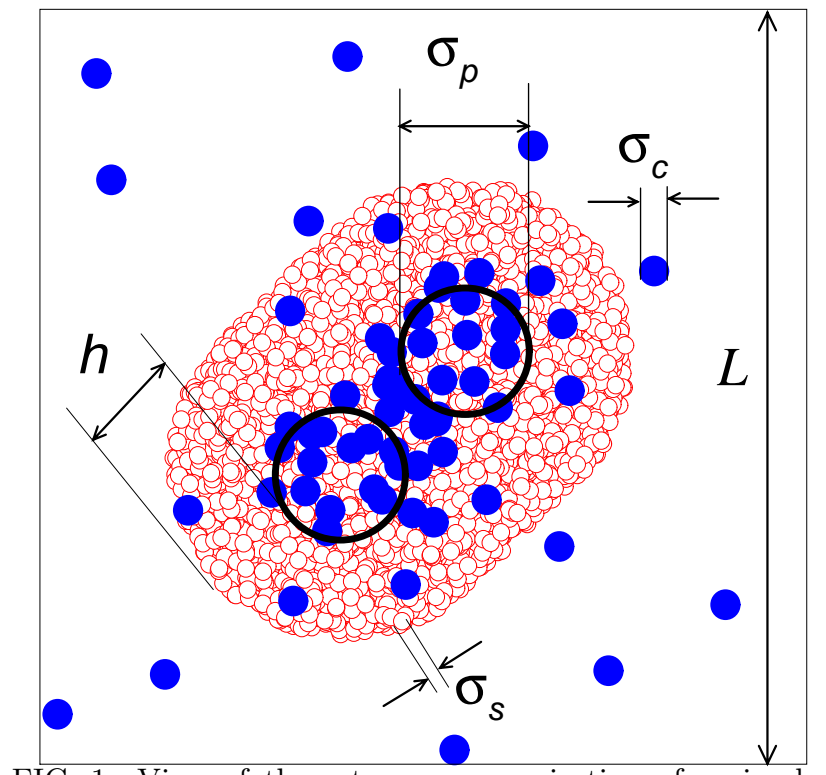

FIG. 1. View of the set-up as a projection of a simulation snapshot: Two polyions (dark open circles) in a bath of solvent particles (small hollow spheres) contained in a spherocylindric cell of width $h$. The counterions shown as small dark spheres can move in the whole simulation box of size $L$.

In this Letter, we investigate the influence of solvent granularity on the effective interactions between charged colloids. We explicitly add to the PM a molecular solvent modelled by a hard sphere fluid. We study this model by direct computer simulation and use the concept of effective interactions to overbridge the gap between microscopic and mesoscopic length scales. Our motivation to do so is twofold: First, although the dipolar nature of the solvent 10 is not included, the model provides a minimal framework towards a statistical description of hydration forces. Second, the solvent hard sphere model was considered in earlier studies for the effective interaction between charged plates using liquid integral equations [11], modified Poisson-Boltzmann theory [12 or more sophisticated density functional approaches [13]. All these descriptions, however, suffer from additional uncontrolled 
approximations such that "exact" computer simulation results are highly desirable. Such simulations were performed for parallel plates [14 and for small neutral particles 15 but are hitherto not available for spherical charged colloids.

We implement a new "solvent bath" simulation scheme which allows to simulate many neutral spheres together with the charged species and obtain explicit results for the effective force between nano-sized highly charged colloids. We use these data to test a theory with solventaveraged effective interactions between the charged particles similar in spirit to the old McMillan-Mayer approach for electrolyte solutions [16]. This solvent-averaged primitive model (SPM) yields good agreement with the simulation data and can thus be used to obtain the effective interaction between larger colloidal particles. For monovalent counterions and large distances $r$, our simulation data can be described perfectly within a Yukawapotential with a solvent-induced polyion charge and screening length renormalization. For divalent counterions and nano-sized colloids, we find an attractive force. Both effects are not contained in the PM.

We consider two large spherical polyions with diameter $\sigma_{p}$ and charge $q_{p}$ at distance $r$, together with their counterions of diameter $\sigma_{c}$ and charge $q_{c}$ in a bath of a neutral solvent $\left(q_{s}=0\right)$ with diameter $\sigma_{s}$. In our model, the pair potentials between the particles as a function of the mutual distances $r$ are a combination of excluded volume and Coulomb terms

$$
V_{i j}(r)= \begin{cases}\infty & \text { for } r \leq\left(\sigma_{i}+\sigma_{j}\right) / 2 \\ q_{i} q_{j} / \epsilon r & \text { else }\end{cases}
$$

where $\epsilon$ is the a smeared background dielectric constant of the solvent and $(i j)=(p p),(p c),(p s),(c c),(c s),(s s)$. Further parameters are the thermal energy $k_{B} T$ and the partial number densities $\rho_{i} \quad(i=p, c, s)$ which can be expressed as partial volume fractions $\phi_{i}=\pi \rho_{i} \sigma_{i}^{3} / 6(i=$ $p, c, s)$. Charge neutrality requires $\rho_{p}\left|q_{p}\right|=\rho_{c}\left|q_{c}\right|$. We fix the two polyions along the body diagonal in a cubic simulation box of length $L$ with periodic boundary conditions, hence $\rho_{p}=2 / L^{3}$. In a dense fluid solvent $\left(\phi_{s} \approx 0.3\right)$, many solvent spheres are in the box, such that a direct simulation is impossible. Thus we resort to the following "solvent-bath" procedure: we define a spherocylindrical cell around the colloidal pair such that the minimal distance $h$ from the colloidal surface to the cell boundary is larger than the hard sphere bulk correlation length $\xi$, see Figure 1 for the set-up. The hard sphere solvent is only contained in this spherocylinder while the counterions can move within the whole simulation box. We use the Molecular Dynamics (MD) method to calculate the particle trajectories. Once a solvent particle is leaving the spherocylindrical cell, it is randomly inserted at another place of the cell boundary with the same velocity and a random depth in order to avoid unphysical solvent layering on the cell surface. Since $h$ is much larger than $\xi$, the presence of the boundary has no influence on the inhomogeneous density distribution of the solvent and the counterions near the colloidal surfaces. For a counterion approaching the cell boundary, however, there is an artificial asymmetry between the solvent bath inside the cell and the "vacuum" outside the cell which hinders a counterion to penetrate into the solvent bath. This unphysical effect is repaired in the simulation scheme by switching off the counterion-solvent interaction for a counterion which is penetrating from outside. Once the counterion is fully surrounded by solvent molecules the interaction is turned on again. This procedure guarantees a symmetric crossing rate of counterions across the spherocylindrical cell. In the solvent-free space outside the cell, the counterion-counterion interaction is still $V_{c c}(r)$ as the mean counterion distance is much larger than $\xi$ such that the Coulomb repulsion dominates solvent depletion effects. The algorithm was carefully tested for solvent slabs between charged plates and perfect agreement was found compared to simulations where the whole space was filled with solvent particles.

In our simulations, we fixed $T=298^{\circ} \mathrm{K}$ and $\epsilon=81$ (water at room temperature) with $\sigma_{s}=3 \AA$, $\phi_{s}=0.3$ (such that $\xi$ is about $3 \sigma_{s}$ ) and $\sigma_{c}=6 \AA$. The width $h$ is $12 \sigma_{s}$ such that typically $N_{s}=25.000-30.000$ solvent hard spheres are simulated. We varied the polyion size $\sigma_{p}$ and charge $q_{p}$ and calculated the solvent- and counterionaveraged total force acting onto a polyion for a given colloidal distance $r$. The force is projected onto the separation vector of the two colloidal spheres such that a positive sign means repulsion. This effective force $F(r)$ is the sum of four different contributions [7]: the direct Coulomb repulsion as embodied in $V_{p p}(r)$, the counterion screening resulting from the averaged Coulomb force of counterions acting onto the polyions, the counterion depletion term arising from the hard sphere part of $V_{p c}(r)$, and the solvent depletion force.

For nano-sized colloids, explicit results for $F(r)$ are presented in Figures $2 \mathrm{a}$ and $2 \mathrm{~b}$. For nearly touching polyions (full curves in the insets of Figs. 2a and 2b) the force exhibits oscillations on the scale of the solvent diameter due to solvent layering leading to attraction for touching polyions as the attractive solvent depletion part exceeds the bare Coulomb repulsion. For larger distances and monovalent counterions, the force is repulsive. Simulation data for the PM are also included which overestimate the force. The repulsion is even stronger in DLVO theory as derived from the Yukawa pair potential

$$
V(r)=\frac{q_{p}^{2} \exp \left(-\kappa\left(r-\sigma_{p}\right)\right)}{\left(1+\kappa \sigma_{p} / 2\right)^{2} \epsilon r}
$$

with $\kappa=\sqrt{4 \pi \rho_{c} q_{c}^{2} / \epsilon k_{B} T}$. For divalent counterions, on the other hand, there is attraction within a range of several polyion diameters. This attraction originates from counterion overscreening induced by hydration, as the pure PM yields repulsive forces.

Intermediate between the PM and the full solvent simulation, we put forward a description on the primitive 

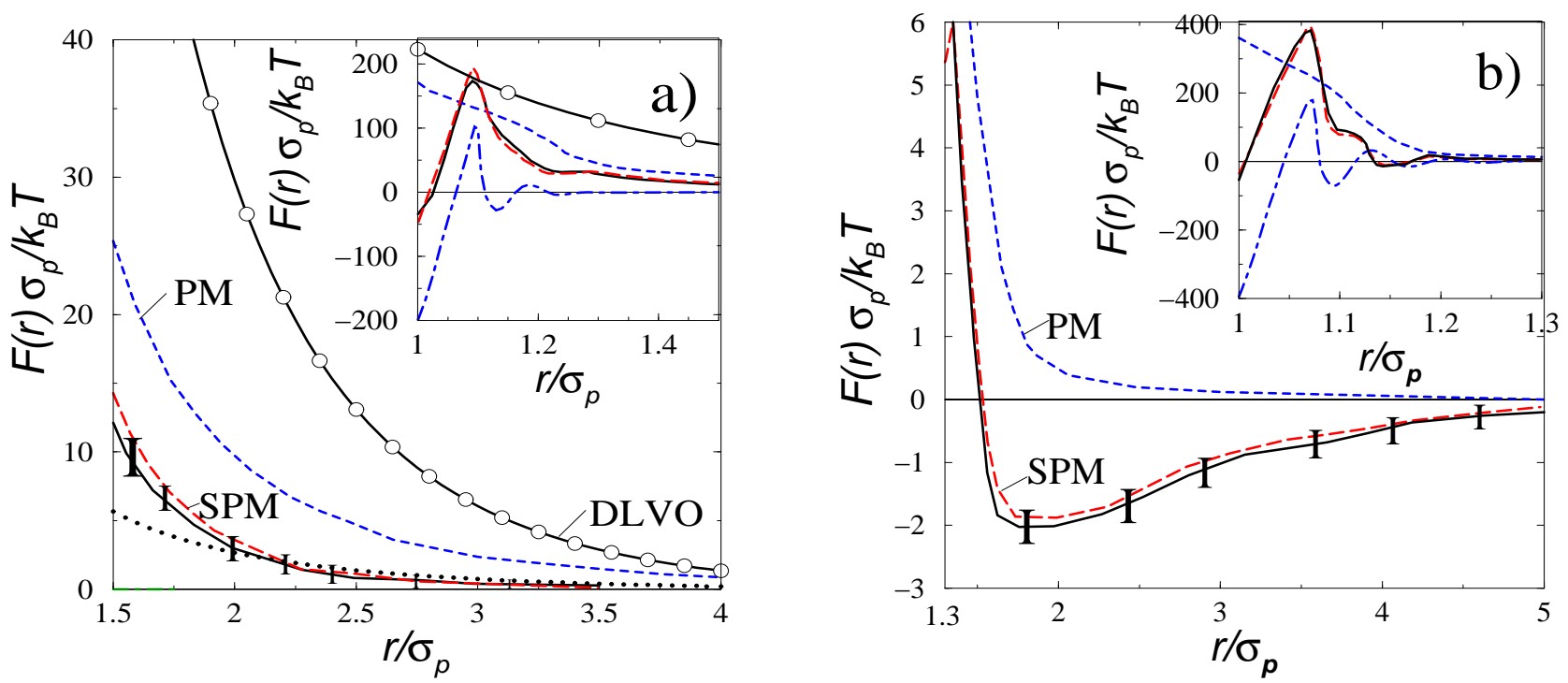

FIG. 2. Reduced distance-resolved force $F(r) \sigma_{p} / k_{B} T$ versus reduced distance $r / \sigma_{p}$. The inset shows the same for nearly touching polyions of molecular distances. a ) for monovalent counterions and $\sigma_{p}: \sigma_{c}: \sigma_{s}=10: 2: 1$; b) for divalent counterions and $\sigma_{p}: \sigma_{c}: \sigma_{s}=14: 2: 1$. The further parameters are $\left|q_{p} / q_{c}\right|=32$ and $\phi_{p}=5.8 \times 10^{-3}$. Solid line with error bars: full solvent bath simulation; long-dashed line: SPM; short-dashed line: PM; open circles: DLVO theory; dot-dashed line in inset: solvent depletion force. The dotted line in a) is the solvent-renormalized Yukawa model.

level with solvent-averaged interactions between the charged particles which provides a simple physical picture of discrete solvent effects. In fact, by tracing out the solvent degrees of freedom exactly, one arrives at additional solvent-induced interactions on the primitive level for which we approximately only consider pairwise terms. This defines the solvent-averaged primitive model (SPM). The additional depletion interactions in hard sphere mixtures have been studied extensively by theory 17] and simulation 18]. The polyion-polyion solvent-induced depletion force is also included in Figure 2 exhibiting oscillations on the scale of the molecular solvent diameter $\sigma_{s}$. While the additional solvent-averaged counterioncounterion interaction is much smaller than the Coulomb repulsion, the solvent-averaged polyion-counterion interaction results in a deep counterion attraction towards the colloidal surfaces with a molecular range and a potential energy depth of several $k_{B} T$, describing granular hydration forces. The only approximation used to derive the SPM is that solvent-induced many-body forces between the charged particles are ignored. This is justified as typical distances between triplets of charged particles are larger than $\xi$ except for nearly touching polyions with "squeezed" counterions. We determine the additional depletion interaction of the SPM by a reference simulation of two spheres in a hard sphere solvent [18] and use them as an input for a simulation of the SPM. As can be deduced from Figure 2, the SPM describes the solvent bath data extremely well, yielding results that lie within the statistical error of the full simulation over the whole range of distances.

We finally use the SPM to investigate solvent effects for polyion sizes in the colloidal domain. Distance-resolved colloidal forces $F(r)$ for monovalent counterions are presented in Figure 3. These forces are repulsive but much smaller than those from PM simulations or DLVO theory. This is due to counterion accumulation near the colloidal surface as induced by the additional solvent depletion attraction. As the corresponding potential energy gain is only few $k_{B} T$, this depletion attraction is different from chemisorption of counterions. For very large distances, on the other hand, the screening of the remaining free counterions will dominate the interaction which can be described by a cell model 汪. To test this, we have performed additional solvent-bath simulations for a single polyion in a spherical cell of radius $R=\left(4 \pi \rho_{p} / 3\right)^{-1 / 3}$ calculating the counterion density $\tilde{\rho}_{c}$ at the cell boundary. The corresponding effective Yukawa potential has the same form as in eq.(2) but with a solvent-renormalized screening length $\kappa^{*}=\kappa \sqrt{\tilde{\rho}_{c} / \rho_{c}}$ and a solvent-renormalized charge $q_{p}^{*}=q_{p} \tilde{\rho}_{c} / \rho_{c}$ which is considerably smaller than the bare charge. The actual value of this renormalized charge, however, differs strongly from the charge renormalization according to the PM or Poisson Boltzmann theory [4]. The force resulting from the solvent-renormalized Yukawa model fits our full simulation data of nano-sized colloids for large distances and monovalent counterions (see Figure 2a) and perfectly describes the SPM data for larger colloids except for molecular distances (see Figure 3). Consequently, a Yukawa model can still be used but the parameters have to be suitably renormalized. Clearly, the repulsive Yukawa model breaks down if no free counterions are left as is the case for, e.g., divalent counterions 
and nano-sized colloids.

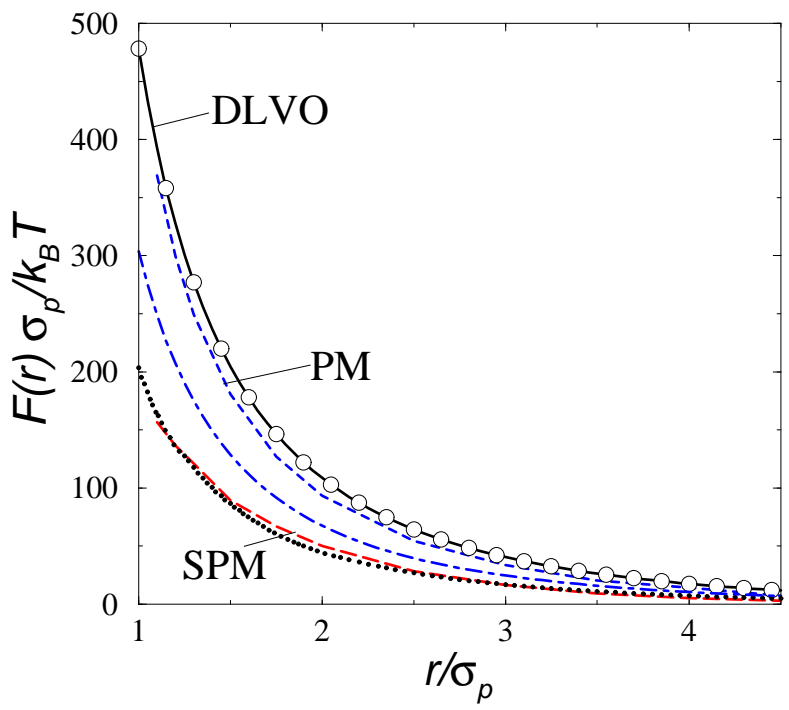

FIG. 3. Same as Fig.2 but now for larger polyions $\sigma_{p}: \sigma_{c}: \sigma_{s}=370: 2: 1,\left|q_{p} / q_{c}\right|=280, \phi_{p}=2.3 \times 10^{-3}$, and monovalent counterions. The dot-dashed line here is the PM with colloidal charge reduction by the corresponding average amount of counterions in a shell of width $\xi$ around the colloids. The meaning of the other lines is as in Figure 2a.

We finally remark that the traditional justification of the PM is to define a bare charge by fixing all the counterions which are in a molecular shell around the colloids and to treat the rest within the PM. This picture of polyion charge reduction works for chemisorption but not for the weaker hydration forces. In fact, if the polyion charge is reduced by the corresponding average counterion charge in a shell of thickness of the solvent bulk correlation length $\xi$, the PM still overestimates the force considerably, see the dot-dashed line Figure 3.

In conclusion, based on a unified statistical description of counterion hydration and screening, we have shown that hydration forces profoundly influence the colloidal interaction. For divalent counterions, there is solventinduced attraction which is not contained in the traditional primitive model but can be encaptured within a solvent-averaged primitive model. For monovalent counterions, the forces can be described by a charge renormalization induced by counterion hydration forces. This picture is in agreement with experiments on strongly deionized samples where a Yukawa picture can still be employed, provided the colloidal charge is renormalized towards a value smaller than the bare charge [5, 19. The general concept of charge renormalization should be transferable to other situations where screening by few remaining "free" counterions dominates the interaction as, e.g., for a polar solvent, for different polyionic surface charge patterns and for explicit surface chemistry.

We thank R. Roth, C.N. Likos and T. Palberg for helpful remarks.
[1] "Structure and Dynamics of Strongly Interacting Colloids and Supramolecular Aggregates in Solution" edited by S.-H. Chen, J. S. Huang, P. Tartaglia, NATO ASI Series, Vol. 369, Kluwer Academic Publishers, Dordrecht, 1992.

[2] J. P. Hansen, H. Löwen, Ann. Rev. Phys. Chemistry, October 2000, in press.

[3] B. V. Derjaguin, L. D. Landau, Acta Physicochim. USSR 14, 633 (1941); E. J. W. Verwey and J. T. G. Overbeek, "Theory of the Stability of Lyophobic Colloids" (Elsevier, Amsterdam, 1948).

[4] S. Alexander, P. M. Chaikin, P. Grant, G. J. Morales, P. Pincus, D. Hone, J. Chem. Phys. 80, 5776 (1984).

[5] W. Härtl, H. Versmold, J. Chem. Phys. 88, 7157 (1988); T. Palberg, W. Mönch, F. Bitzer, R. Piazza, T. Bellini, Phys. Rev. Lett. 74, 4555 (1995).

[6] J. C. Crocker, D. G. Grier, Phys. Rev. Lett. 73, 352 (1994); G. M. Kepler, S. Fraden, Phys. Rev. Lett. 73, 356 (1994); D. G. Grier, Nature (London) 393, 621 (1998).

[7] E. Allahyarov, I. D'Amico, H. Löwen, Phys. Rev. Lett. 81, 1334 (1998).

[8] P. Linse, V. Lobaskin, Phys. Rev. Lett. 83, 4208 (1999).

[9] R. Messina, C. Holm, K. Kremer, Phys. Rev. Lett. 85, 872 (2000).

[10] M. Kinoshita, S. Iba, M. Harada, J. Chem. Phys. 105, 2487 (1996).

[11] F. Otto, G. N. Patey, Phys. Rev. E 60, 4416 (1999); J. Chem. Phys. 112, 8939 (2000).

[12] V. Kralj-Iglic, A. Iglic, J. Physique II (France) 6, 477 (1996); J. Borukhov, D. Andelman, H. Orland, Phys. Rev. Lett. 79, 435 (1997); Y. Burak, D. Andelman, to be published; E. Trizac, J.-L. Raimbault, condmat/9909420.

[13] Z. Tang, L. E. Scriven, H. T. Davis, J. Chem. Phys. 100, 4527 (1994); L. J. D. Frink, F. van Swol, J. Chem. Phys. 105, 2884 (1996); T. Biben, J. P. Hansen, Y. Rosenfeld, Phys. Rev. E 57, R3727 (1998) C. N. Patra, J. Chem. Phys. 111, 9832 (1999); D. Henderson, P. Bryk, S. Sokolowski, D. T. Wasan, Phys. Rev. E 61, 3896 (2000).

[14] See e.g.: D. Boda, D. Henderson, J. Chem. Phys. 112, 8934 (2000).

[15] J. Rescic, V. Vlachy, L. B. Bhuiyan, C. W. Outhwaite, J. Chem. Phys. 107, 3611 (1997).

[16] W. G. McMillan, J. E. Mayer, J. Chem. Phys. 13, 276 (1945).

[17] M. Dijkstra, R. van Roij, R. Evans, Phys. Rev. Lett. 81, 2268 (1998); B. Götzelmann, R. Roth, S. Dietrich, M. Dijkstra, R. Evans, Europhys. Lett. 47, 398 (1999).

[18] R. Dickman, P. Attard, V. Simonian, J. Chem. Phys. 107, 205 (1997); M. Dijkstra, R. van Roij, R. Evans, Phys. Rev. Lett. 82, 117 (1999).

[19] T. Gisler, S. F. Schulz, M. Borkovec, H. Sticher, P. Schurtenberger, B. D'Aguanno, R. Klein, J. Chem. Phys. 101, 9924 (1994). 